\title{
Comparison of visual and refractive outcomes between femtosecond laser-assisted in situ keratomileusis (FS-LASIK) and photorefractive keratectomy (PRK): a long-term outcomes analysis
}

\author{
@Mehmet Fatih Karadağ \\ Dünyagöz Hospital, Department of Ophthalmology, Gaziantep, Turkey
}

Cite this article as: Karadağ MF. Comparison of visual and refractive outcomes between femtosecond laser-assisted in situ keratomileusis (FS-LASIK) and photorefractive keratectomy (PRK): a long-term outcomes analysis. J Health Sci Med 2022; 5(1): 257-261.

\begin{abstract}
Aim: We aimed to compare the results of photorefractive keratectomy (PRK) and femtosecond-assisted laser in situ keratomileusis (FS-LASIK) procedures in the treatment of myopia and myopic astigmatism.

Material and Method: Seventy eyes of 35 patients with myopia and/or myopic astigmatism who had undergone PRK procedure were compared retrospectively with 70 eyes of 35 patients with myopia and/or myopic astigmatism who had undergone FSLASIK procedure.

Results: All patients completed the 2-year follow-up period. With respect to age and sex, PRK and FS-LASIK groups were comparable. The differences in uncorrected distance visual acuity (UDVA), corrected distance visual acuity (CDVA), and spherical equivalent were significantly better with PRK than with FS-LASIK at 6- and 24-month visits. FS-LASIK and PRK had similar values of safety index, efficacy index, and predictability at 6 and 24 months postoperatively. No significant complications were observed in neither of the procedures during the follow-up period.

Conclusion: Both PRK and FS-LASIK seem equally effective options for the correction of myopia and myopic astigmatism. However, PRK provided slightly better visual and refractive outcomes than FS-LASIK at 6 and 24 months postoperatively.
\end{abstract}

Keywords: Laser in situ keratomileusis, myopia, myopic astigmatism, photorefractive keratectomy, refractive surgery

\section{INTRODUCTION}

Two frequently utilized types of refractive surgery, one of the most frequently performed elective procedures in ophthalmic surgery practice, are photorefractive keratectomy (PRK) and laser-assisted in situ keratomileusis (LASIK). On the one hand, PRK is a surface ablation procedure in which the epithelial layer of the cornea is removed, followed by laser ablation applied to the corneal stroma to change the refractive power (1). On the other, femtosecond laser-assisted LASIK (FS-LASIK) uses infrared light $(1,053 \mathrm{~nm})$ to produce microplasma and microcavitation bubbles within the corneal stroma and thereby functionally create a corneal dissection plane interface that can be manually opened with minimal effort (2).

Since its introduction in 2001, femtosecond laser technology has continued to evolve and even become the preferred method for flap creation in most LASIK operations (3). The rapid improvement in vision and lack of postoperative pain with LASIK has also made it preferred by patients over PRK, which causes greater postoperative discomfort and prolongs the recovery of visual acuity (4). On the other hand, PRK eliminates flap-related complications and may be associated with a decreased incidence of postoperative dry eye. Beyond that, in response to PRK's major complicationscorneal haze and regression in patients with highdiopter (D) - use of mitomycin-C (MMC) was devised to decrease the development of major complications (5). Studies have also shown favorable visual and refractive outcomes with PRK-MMC used to treat cases of high myopia in the short- and long-term $(6,7)$.

Although many studies have evaluated the efficacy and safety of FS-LASIK and PRK in isolation (8), to our knowledge, few studies compared the long-term results 
of patients who underwent either PRK or FS-LASIK (9, 10). Thus, the aim of the present study was to compare visual and refractive outcomes in patients 24 months after they received PRK or FS-LASIK to treat myopia or myopic astigmatism.

\section{MATERIAL AND METHOD}

The study protocol was approved by Dünyagöz Hospital Ethics Committee (Date: 16.09.2021, Decision No: 2021/833). All procedures were performed adhered to the ethical rules and principles of the Helsinki Declaration.

\section{Eyes and the Setting}

This retrospective, comparative study was performed by reviewing the charts of patients with varying severity of myopia and myopic astigmatism who underwent FS-LASIK or PRK at a private ophthalmology clinic in Turkey. All of the surgical procedures were performed by the same ophthalmic surgeon (MFK). To ensure equal conditions, operated eyes were selected in consecutive patients by turn. Exclusion criteria were as follows: the presence of concurring ocular or systemic disease, unstable refractive error, manifest or suspected corneal ectatic disorders, history of herpetic keratitis or corneal dystrophy, corneal scarring, cataract, glaucoma, pregnancy, and active use of isotretinoin or hormonal therapy. Contact lenses were discontinued 2 weeks prior to screening for soft contact lens wearers and 6 weeks prior to screening for rigid gas permeable lens wearers.

\section{Pre- and Post-operative Assessments}

All patients underwent a preoperative examination involving assessment of uncorrected distance visual acuity (UDVA) and corrected distance visual acuity (CDVA), intraocular pressure measurement, slit-lamp examination of the anterior segment, and dilated fundus examination at initial preoperative evaluation. All assessments were repeated postoperatively on day 1 , at week 1 and at months 1, 3, 6, 12, and 24 .

Visual acuity was recorded in both Snellen notation and logarithm of the minimum angle of resolution (logMAR) format. The efficacy index was defined as the ratio of postoperative UDVA to preoperative CDVA. The safety index was defined as the ratio of mean postoperative CDVA to mean preoperative CDVA. Predictability was presented as the percentage of eyes within $\pm 0.50 \mathrm{D}$ postoperatively. They were calculated separately for the FS-LASIK and PRK groups.

\section{Surgical Procedures}

PRK was offered to patients whose central pachymetry was less than $500 \mu \mathrm{m}$ (i.e., PRK Group), whereas
FS-LASIK was offered to patients whose central pachymetry exceeded $500 \mu \mathrm{m}$ (i.e., FS-LASIK Group). In using both techniques, a residual stromal bed thickness of more than $300 \mu \mathrm{m}$ was preserved. The target refraction in all patients was emmetropia.

In the PRK group, the epithelial layer of the anesthetized (proparacaine hydrochloride $0.5 \%$ ) cornea was removed mechanically. The VISX STAR S4 Excimer Laser (VISX Inc., Santa Clara, CA, USA) was used for corneal ablation, performed with a $1.25-\mathrm{mm}$ blend zone and a $6.5-\mathrm{mm}$ ablation zone. Following ablation, $0.02 \%$ MMC was applied to the stromal bed for 30 seconds to prevent scarring and haze, after which the bed was washed with a balanced salt solution to remove any debris. After irrigation with $30 \mathrm{~mL}$ of balanced salt solution, a bandage contact lens (Air Optix, CIBA VISION) was fitted over the treated cornea for 4 days. After the surgical procedure, each patient was prescribed a topical steroid (dexamethasone sodium phosphate $0.1 \%) 4$ times daily for 3 weeks, a topical antibiotic (besifloxacin $0.6 \%$ ) thrice daily for a week, nepafenac $0.1 \%$ thrice daily for 3 weeks, and preservative-free artificial tears (trehalose $3 \%$, sodium hyaluronate $0.15 \%) 5$ times daily for 3 months. Each patient was examined on a daily basis until the epithelium healed completely.

In the FS-LASIK group, following topical anesthesia, a $110-\mu \mathrm{m}$ thick flap was attempted using the $150-\mathrm{kHz}$ IntraLase iFS (Abbott Medical Optics, Santa Ana, CA, USA) femtosecond laser platform. After lifting the flap, corneal ablation was performed using the VISX STAR S4 Excimer Laser (VISX Inc., Santa Clara, CA, USA), with a $6.5-\mathrm{mm}$ optical zone and $1.25-\mathrm{mm}$ blend zone. Following irrigation with a balanced saline solution, the flap was repositioned. Postoperative treatment was the same as in the PRK group.

\section{Statistical Analysis}

All statistical analyses were performed using the SPSS statistical software version 25.0 (IBM, Armonk, NY, USA). For each variable, the normality of the data distribution was evaluated using the KolmogorovSmirnov test. The normality test showed that each numerical variable was normally distributed. All values were reported as the number ( $\mathrm{n}$ ) or mean \pm standard deviation. The categorical data were analyzed using the chi-square test. The Independent Samples t-test was used to compare the variables between the FSLASIK and PRK groups. One-way analysis of variance (ANOVA) was used to analyze timely changes postoperatively, and Dunnett's test was used for multiple comparisons. A value of $\mathrm{p}<0.05$ was defined for statistical significance. 


\section{RESULTS}

The study included 140 eyes of 70 patients with myopia and/or myopic astigmatism, half of whom received FSLASIK ( $n=70$ eyes, Group 1), whereas the other half received PRK ( $\mathrm{n}=70$ eyes, Group 2), between September 2018 and September 2020. The demographic, as well as preoperative and intraoperative clinical characteristics of the patients, are summarized in Table 1. FS-LASIK group contained 17 men and 18 women with a mean age of $28.14 \pm 6.03$ years, PRK group contained 18 men and 17 women with a mean age of $26.51 \pm 6.05$ years. The distribution of patients by age and gender in the FS-LASIK group versus the PRK group did not differ significantly $(\mathrm{P}=.113$ and $\mathrm{P}=.951$, respectively). Furthermore, preoperative clinical parameters regarding central corneal thickness, spherical equivalent, astigmatism, average keratometric value, UDVA, and CDVA were similar between the groups.

All postoperative follow-up visits were completed in both groups. No severe complications were observed during the follow-up periods, such as corneal ectasia, epithelial ingrowth, diffuse lamellar keratitis, transient photosensitivity syndrome, severe dry eye, or keratitis.

The changes in average visual acuity and refraction parameters are summarized in Table 2. UDVA, CDVA, spherical equivalent, and cylindrical refraction values were successfully improved in both groups at the end of 24 months ( $p<0.001$, for all). The differences in UDVA, CDVA, and spherical equivalent were significantly slightly better in the PRK group than in FS-LASIK at 6- and 24-months follow-up. No statistically significant differences were observed in cylindrical refraction between the groups 6 and 24 months after surgery.

Table 3 shows the comparison of safety index, efficacy index, and predictability values between the groups. FS-LASIK and PRK have similar values of safety index, efficacy index, and predictability at 6 and 24 months postoperatively.

\section{DISCUSSION}

The main findings of the present study were as follows: (i) Both PRK and FS-LASIK achieved the treatment goal, the visual outcomes of PRK were slightly better than FS-LASIK at 24 months, though. (ii) No intraoperative or postoperative complications arose in any of the study participants. (iii) Ours was among the studies with the longest follow-up duration in comparison of PRK vs. FS-LASIK.

Studies with long-term follow-up periods to compare PRK with flap-based procedures have been few and far between. Steinert et al. (11) who compared the outcomes of LASIK and PRK in patients with myopia, found
Table 1. The demographic and preoperative clinical characteristics

\begin{tabular}{|lccc|}
\hline Parameters & WFG FS-LASIK & PRK & p values \\
\hline Age (Years) & $28.14 \pm 6.03$ & $26.51 \pm 6.05$ & $0.113^{\mathrm{a}}$ \\
Gender $($ male/female) & $17 / 18$ & $18 / 17$ & $0.951^{\mathrm{b}}$ \\
CCT $(\mu \mathrm{m})$ & $534.12 \pm 31.42$ & $539.25 \pm 28.12$ & $0.457^{\mathrm{a}}$ \\
SE $(\mathrm{D})$ & $-3.25 \pm 1.65$ & $-3.18 \pm 1.72$ & $0.256^{\mathrm{a}}$ \\
Astigmatism $(\mathrm{D})$ & $-0.84 \pm 0.75$ & $-0.91 \pm 0.71$ & $0.574^{\mathrm{a}}$ \\
K average $(\mathrm{D})$ & $43.74 \pm 1.51$ & $43.53 \pm 1.42$ & $0.321^{\mathrm{a}}$ \\
UDVA (LogMAR) & $1.25 \pm 0.20$ & $1.22 \pm 0.21$ & $0.725^{\mathrm{a}}$ \\
CDVA (LogMAR) & $-0.01 \pm 0.04$ & $-0.01 \pm 0.05$ & $0.856^{\mathrm{a}}$
\end{tabular}

Abbreviations: D; diopter, CCT; central corneal thickness, SE; spherical equivalent, $\mathrm{K}$; keratometry, UDVA; uncorrected distance visual acuity, CDVA; corrected distance visual acuity. Values are expressed as $\mathrm{n}$ or mean \pm standard deviation. a Independent sample test, b Chi-squared test.

Table 2. The preoperative and postoperative findings of patients

\begin{tabular}{|ccccc|}
\hline Groups & $\begin{array}{c}\text { UDVA } \\
\text { (logMAR) }\end{array}$ & $\begin{array}{c}\text { CDVA } \\
\text { (logMAR) }\end{array}$ & $\begin{array}{c}\text { Spherical } \\
\text { Equivalent } \\
\text { (D) }\end{array}$ & $\begin{array}{c}\text { Cylindrical } \\
\text { Refraction } \\
\text { (D) }\end{array}$ \\
\hline WFG FS-LASIK & $(\mathrm{n}=70)$ & & & \\
Preoperative & $1.25 \pm 0.20$ & $-0.01 \pm 0.04$ & $-3.25 \pm 1.65$ & $-0.84 \pm 0.75$ \\
6 months & $0.01 \pm 0.07$ & $-0,03 \pm 0.04$ & $-0.37 \pm 0.26$ & $-0.31 \pm 0.33$ \\
24 months & $-0.01 \pm 0.05$ & $-0.06 \pm 0.03$ & $-0.27 \pm 0.23$ & $-0.09 \pm 0.21$ \\
p values a & $<0.001$ & $<0.001$ & $<0.001$ & $<0.001$ \\
PRK (n=70) & & & & \\
Preoperative & $1.22 \pm 0.21$ & $-0.01 \pm 0.05$ & $-3.18 \pm 1.72$ & $-0.91 \pm 0.71$ \\
6 months & $-0.04 \pm 0.07$ & $-0.07 \pm 0.05$ & $-0.21 \pm 0.34$ & $-0.28 \pm 0.27$ \\
24 months & $-0.08 \pm 0.06$ & $-0.11 \pm 0.04$ & $-0.11 \pm 0.23$ & $-0.12 \pm 0.22$ \\
p values a & $<0.001$ & $<0.001$ & $<0.001$ & $<0.001$ \\
p values b between groups & & & \\
Preoperative & 0.725 & 0.856 & 0.256 & 0.574 \\
6 months & 0.009 & 0.005 & 0.004 & 0.512 \\
24 months & 0.001 & 0.002 & 0.003 & 0.445 \\
\hline
\end{tabular}

Abbreviations: D; diopter, UDVA; uncorrected distance visual acuity, CDVA; corrected distance visual acuity. Values are expressed as mean \pm standard deviation. a Repeated measured test, b Independent sample test

Table 3. Comparison of Safety Index, Efficacy Index, and Predictability values between the groups

\begin{tabular}{|c|c|c|c|}
\hline & $\begin{array}{c}\text { WFG FS-LASIK } \\
(\mathbf{n = 7 0 )}\end{array}$ & PRK $(n=70)$ & $p$ values $^{a}$ \\
\hline \multicolumn{4}{|l|}{ Safety Index } \\
\hline 6 months & $1.11 \pm 0.06$ & $1.12 \pm 0.07$ & 0.398 \\
\hline 24 months & $1.10 \pm 0.06$ & $1.13 \pm 0.04$ & 0.431 \\
\hline \multicolumn{4}{|l|}{ Efficacy Index } \\
\hline 6 months & $1.08 \pm 0.11$ & $1.09 \pm 0.12$ & 0.312 \\
\hline 24 months & $1.07 \pm 0.11$ & $1.08 \pm 0.10$ & 0.362 \\
\hline \multicolumn{4}{|c|}{ Predictability (\%) } \\
\hline 6 months & 92.93 & 94.43 & 0.192 \\
\hline 24 months & 92.03 & 95.48 & 0.179 \\
\hline
\end{tabular}

similar efficacy indices between the groups at 12 months, although the improvement in UDVA was more rapid with LASIK than with PRK. Also, in that study, patients who underwent LASIK showed a tendency toward undercorrection more than patients who underwent PRK (11). 
Similarly, in a randomized clinical trial comparing PRK and LASIK, Hersh et al. (12) observed similar efficacy indices between the groups at 12 months, although patients who underwent LASIK again displayed a greater tendency toward under-correction than patients who underwent PRK.

By contrast, some studies have revealed slighter higher efficacy in eyes treated with PRK than with LASIK $(13,14)$. Wallau and colleagues (15) compared LASIK and PRK with MMC and found that UDVA was significantly higher in patients who underwent PRK with MMC than in ones who underwent LASIK both at 3 and 6 months after surgery. In another clinical trial comparing PRK with mechanical epithelial removal, transepithelial PRK, laser-assisted subepithelial keratectomy (LASEK), and LASIK, visual outcomes at postoperative 12 months were slightly better in patients who underwent PRK with mechanical epithelial removal and transepithelial PRK than in their counterparts who underwent LASIK or LASEK (16). Aslanides et al. (17) additionally found that single-step modified transepithelial PRK and conventional alcoholassisted PRK provided significantly better UDVA than LASIK for patients with myopia of $6.00 \mathrm{D}$ or more at 12 months. In contrast to those studies, however, Van Gelder et al. (18) reported that in patients with mild to moderate myopia, flap-based surgeries provided better visual and more predictable refractive outcomes than PRK-based surgeries.

In line with the previous studies in the literature, we found slightly better UDVA, CDVA, and spherical equivalent values in patients who underwent PRK than in ones who underwent FS-LASIK at 6 months. Moreover, that difference remained statistically significant 24 months after the procedures, and the safety index, efficacy index, and predictability were all similar in both groups at 6 and 24 months postoperatively. Although other researchers found that the rate of reported complications developing in patients who underwent LASIK was higher than in ones who underwent PRK (16), we observed no complications in either group in our study. Therefore, we do not think that the difference in UDVA was due to a high rate of complications in FS-LASIK. In our opinion, the main reason for this may be due to the high-order aberrations triggered in patients who received FS-LASIK and structural changes caused by the creation of a corneal flap. Indeed, past studies have demonstrated that higher-order optical aberrations and systematic changes in corneal topography can be associated with uncomplicated lamellar flap creation $(19,20)$.
The major limitation of our retrospective evaluation was the lack of any assessment of contrast sensitivity, corneal aberrations, and other corneal biomechanics. The absence of those additional corneal tests limited our ability to determine the true cause of the difference in visual acuity with either surgical modality.

\section{CONCLUSION}

The present study is one of the few studies with the longest follow-up duration for postoperative outcomes. Our study revealed that PRK and FS-LASIK were equally effective procedures for correcting myopia and astigmatism. Though statistically insignificant, PRK provided slightly better visual and refractive outcomes than FS-LASIK at 6 and 24 months postoperatively.

\section{ETHICAL DECLARATIONS}

Ethics Committee Approval: The study protocol was approved by Dünyagöz Hospital Ethics Committee (Date: 16.09.2021, Decision No: 2021/833).

Informed Consent: Because the study was designed retrospectively, no written informed consent form was obtained from patients.

Referee Evaluation Process: Externally peer-reviewed.

Conflict of Interest Statement: The authors have no conflicts of interest to declare.

Financial Disclosure: The authors declared that this study has received no financial support.

Author Contributions: All of the authors declare that they have all participated in the design, execution, and analysis of the paper, and that they have approved the final version.

\section{REFERENCES}

1. Srinivasan R. Ablation of polymers and biological tissue by ultraviolet lasers. Science (New York, NY). 1986; 234: 559-65.

2. Chen S, Feng Y, Stojanovic A, Jankov MR, 2 ${ }^{\text {nd }}$, Wang Q. IntraLase femtosecond laser vs mechanical microkeratomes in LASIK for myopia: a systematic review and meta-analysis. J Refract Surg (Thorofare, NJ : 1995) 2012; 28: 15-24.

3. Kymionis GD, Kankariya VP, Plaka AD, Reinstein DZ Femtosecond laser technology in corneal refractive surgery: a review. J Refract Surg (Thorofare, NJ: 1995) 2012; 28: 912-20.

4. Shortt AJ, Bunce C, Allan BD. Evidence for superior efficacy and safety of LASIK over photorefractive keratectomy for correction of myopia. Ophthalmology 2006; 113: 1897-908.

5. Majmudar PA, Forstot SL, Dennis RF, et al. Topical mitomycin-C for subepithelial fibrosis after refractive corneal surgery. Ophthalmology 2000; 107: 89-94.

6. Miraftab M, Hashemi H, Asgari S. Matched optical quality comparison of 3-year results of PRK-MMC and phakic IOL implantation in the correction of high myopia. Eye (London, England) 2015; 29: 926-31.

7. Hashemi H, Miraftab M, Asgari S. Comparison of the visual outcomes between PRK-MMC and phakic IOL implantation in high myopic patients. Eye (London, England) 2014; 28: 1113-8. 
8. Rajan MS, Jaycock P, O'Brart D, Nystrom HH, Marshall J. A longterm study of photorefractive keratectomy; 12-year follow-up. Ophthalmology 2004; 111: 1813-24.

9. Castro-Luna G, Jimenez-Rodriguez D, Perez-Rueda A, AlaskarAlani H. Long Term Follow-Up Safety and Effectiveness of Myopia Refractive Surgery. Int J Environ Res Public Health 2020; 17.

10. Taneri S, Knepper J, Rost A, Dick HB. [Long-term outcomes of PRK, LASIK and SMILE]. Ophthalmologe 2021.

11.Steinert RF, Hersh PS. Spherical and aspherical photorefractive keratectomy and laser in-situ keratomileusis for moderate to high myopia: two prospective, randomized clinical trials. Summit technology PRK-LASIK study group. Trans Am Ophthalmol Soc 1998; 96: 197-221.

12.Hersh PS, Brint SF, Maloney RK, et al. Photorefractive keratectomy versus laser in situ keratomileusis for moderate to high myopia. A randomized prospective study. Ophthalmology. 1998; 105: 1512-22.

13. Alió JL, Ortiz D, Muftuoglu O, Garcia MJ. Ten years after photorefractive keratectomy (PRK) and laser in situ keratomileusis (LASIK) for moderate to high myopia (control-matched study). Br J Ophthalmol 2009; 93: 1313-8.

14. Pallikaris IG, Siganos DS. Excimer laser in situ keratomileusis and photorefractive keratectomy for correction of high myopia. J Refract Corneal Surg 1994; 10: 498-510.

15. Wallau AD, Campos M. Photorefractive keratectomy with mitomycin $\mathrm{C}$ versus LASIK in custom surgeries for myopia: a bilateral prospective randomized clinical trial. J Refract Surg (Thorofare, NJ : 1995) 2008; 24: 326-36.

16. Ghadhfan F, Al-Rajhi A, Wagoner MD. Laser in situ keratomileusis versus surface ablation: visual outcomes and complications. J Cataract Refract Surg 2007; 33: 2041-8.

17. Aslanides IM, Georgoudis PN, Selimis VD, Mukherjee AN Single-step transepithelial ASLA (SCHWIND) with mitomycin-C for the correction of high myopia: long term follow-up. Clin Ophthalmol (Auckland, NZ) 2015; 9: 33-41.

18. Van Gelder RN, Steger-May K, Yang SH, Rattanatam T, Pepose JS. Comparison of photorefractive keratectomy, astigmatic PRK, laser in situ keratomileusis, and astigmatic LASIK in the treatment of myopia. J Cataract Refract Surg 2002; 28: 462-76.

19. Chhadva P, Cabot F, Galor A, Karp CL, Yoo SH. Long-term outcomes of flap amputation After LASIK. J Refract Surg (Thorofare, NJ: 1995) 2016; 32: 136-7.

20.Zhao W, Zhang Y. Comparison and predictive factors analysis for efficacy and safety of Kirschner wire, anatomical plate fixation and cannulated screw in treating patients with open calcaneal fractures. Medicine 2019; 98: e17498. 